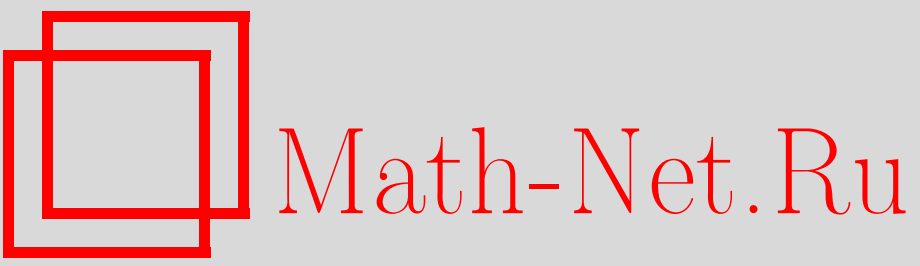

Е. П. Иванова, Дифференциально-разностные уравнения с несоизмеримыми сдвигами аргументов, Итоги науки и техн. Сер. Соврем. мат. и ее прил. Темат. обз., 2021, том 191, 92-100

DOI: https://doi.org/10.36535/0233-6723-2021-191-92-100

Использование Общероссийского математического портала Math-Net.Ru подразумевает, что вы прочитали и согласны с пользовательским соглашением

http://www.mathnet.ru/rus/agreement

Параметры загрузки:

IP: 3.85 .73 .92

26 апреля 2023 г., $17: 11: 03$ 
ИТОГИ НАУКИ И ТЕХНИКИ.

Современная математика и ее приложения.

Тематические обзоры.

Том 191 (2021). C. 92-100

DOI: 10.36535/0233-6723-2021-191-92-100

УДК 517.9

\title{
ДИФФЕРЕНЦИАЛЬНО-РАЗНОСТНЫЕ УРАВНЕНИЯ С НЕСОИЗМЕРИМЫМИ СДВИГАМИ АРГУМЕНТОВ
}

\author{
(c) 2021 г. $\quad$ Е. П. ИВАНОВА
}

\begin{abstract}
АннотАция. Рассматриваются краевые задачи для дифференциально-разностных уравнений с возмущениями в сдвигах аргументов. Получены необходимые и достаточные условия выполнения неравенства типа Гординга в алгебраическом виде. Исследована непрерывная зависимость решений этих задач от сдвигов аргументов.
\end{abstract}

Ключевые слова: краевая задача, дифференциально-разностное уравнение, сильная эллиптичность, неравенство Гординга.

\section{DIFFERENTIAL-DIFFERENCE EQUATIONS WITH INCOMMENSURABLE SHIFTS OF ARGUMENTS}

\author{
(c) 2021 E. P. IVANOVA
}

\begin{abstract}
Boundary-value problems for differential-difference equations with perturbations in shifts of arguments are considered. Necessary and sufficient conditions for the fulfillment of Gårding-type inequalities in algebraic form are obtained. Continuous dependence of solutions of these problems on shifts of arguments is examined.
\end{abstract}

Keywords and phrases: boundary-value problem, differential-difference equation, strong ellipticity, Gårding inequality.

AMS Subject Classification: $47 \mathrm{G} 40$

1. Введение. Основы теории эллиптических функционально-дифференциальных уравнений построены в $[2,6,7]$. Интерес к этим задачам связан с важными приложениями к теории многослойных пластин и оболочек (см. [1,7]), к теории нелинейных лазерных систем (см. [8]), к нелокальным эллиптическим задачам, возникающим в теории плазмы [5] и др. Наиболее глубоко были изучены краевые задачи для эллиптических дифференциально-разностных уравнений с целочисленными или соизмеримыми сдвигами пространственных переменных, содержащихся в разностных операторах. Такие задачи обладают рядом принципиально новых свойств. Например, символ сильно эллиптического дифференциально-разностного оператора может быть отрицательным, а гладкость обобщенных решений соответствующей краевой задачи может нарушаться внутри области и сохраняться лишь в некоторых подобластях. Дифференциально-разностные уравнения с несоизмеримыми сдвигами в одномерном случае рассматривались в $[4,7]$. Новым направлением в теории функционально-дифференциальных уравнений является теория эллиптических функционально-дифференциальных уравнений с растяжением и сжатием пространственных переменных (см. [5]). В частности, там рассматривался вопрос о непрерывной зависимости константы эллиптичности и решений функционально-дифференциальных уравнений от параметра

Работа выполнена при поддержке Российского фонда фундаментальных исследований (проект № 17-01-00401а). 
$\varepsilon$ при $\varepsilon \rightarrow 0$. В настоящей работе автор исследует эллиптические дифференциально-разностные уравнения, содержащие как слагаемые со сдвигами аргументов, независимые от малого параметра, так и слагаемые, содержащие сдвиги, зависимые от $\varepsilon$. Получены необходимые и достаточные условия сильной эллиптичности рассматриваемых уравнений.

2. Сильная эллиптичность семейства дифференциально-разностных операторов с малыми сдвигами. Рассмотрим дифференциально-разностное уравнение

$$
\begin{gathered}
\sum_{|\alpha|,|\beta| \leqslant m} D^{\alpha} R_{\alpha \beta} D^{\beta} u(x)=f(x) \quad(x \in Q), \\
u(x)=0 \quad(x \notin Q),
\end{gathered}
$$

где $Q$-ограниченная область в $\mathbb{R}^{n}$ с кусочно гладкой границей, Разностные операторы $R_{\alpha \beta}: L_{2}\left(\mathbb{R}^{n}\right) \rightarrow L_{2}\left(\mathbb{R}^{n}\right)$ имеют вид

$$
R_{\alpha \beta} u(x)=\sum_{k=-J}^{J} a_{\alpha \beta}^{k} u\left(x_{1}+k \varepsilon, x_{2}, \ldots, x_{n}\right) \quad\left(\varepsilon>0, a_{\alpha \beta}^{k} \in \mathbb{C}\right) .
$$

Назовем уравнение (1) сильно эллиптическим, если выполнено неравенство Гординга:

$$
\operatorname{Re} \sum_{|\alpha|,|\beta| \leqslant m}\left(R_{\alpha \beta} D^{\beta} u, D^{\alpha} u\right)_{L_{2}(G)} \geqslant c_{1}\|u\|_{H^{m}(Q)}^{2}-c_{2}\|u\|_{L_{2}(Q)}^{2} \quad\left(\forall u \in C_{0}^{\infty}(G), c_{1}>0\right) .
$$

Через $H^{m}(Q)$ обозначено пространство Соболева комплекснозначных функций, принадлежащих $L_{2}(Q)$ вместе с обобщенными производными до порядка $m$ включительно. Пространство $H^{m}(Q)$ гильбертово со скалярным произведением

$$
(u, v)_{H^{m}(Q)}=\sum_{|\alpha| \leqslant m} \int_{Q} D^{\alpha} u(x) \overline{D^{\alpha} v(x)} d x
$$

где $\alpha=\left(\alpha_{1}, \ldots, \alpha_{n}\right)$ - мультииндекс, $|\alpha|=\alpha_{1}+\ldots+\alpha_{n}, \alpha_{i} \geqslant 0, D^{\alpha}=\left(-i \partial / \partial x_{1}\right)^{\alpha_{1}} \ldots \cdot\left(-i \partial / \partial x_{n}\right)^{\alpha_{n}}$.

Пространство $\stackrel{\circ}{H}^{m}(Q)$ - замыкание пространства $C_{0}^{\infty}(Q)$ финитных бесконечно дифференцируемых функций в $H^{m}(Q)$. Известно, что в пространстве $\stackrel{\circ}{H}^{1}(Q)$ можно ввести эквивалентное скалярное произведение

$$
(u, v)_{H^{m}(Q)}^{\prime}=\sum_{|\alpha|=m} \int_{Q} D^{\alpha} u(x) \overline{D^{\alpha} v(x)} d x .
$$

Пространство $\stackrel{\circ}{H}^{m}(Q)$ можно отождествить с подпространством функций из $H^{m}\left(\mathbb{R}^{n}\right)$, равных нулю вне $Q$.

Проблема равномерной (по параметру $\varepsilon>0$ ) сильной эллиптичности уравнений (1) на цилиндрической области $\Omega\left(\Omega=(0, a) \times D\right.$, где $D$-ограниченная область в $\left.\mathbb{R}^{n-1}\right)$ исследована Л. Е. Россовским в [5] с помощью предложенного им символа $a_{R}(\eta, \xi)$ :

$$
a_{R}(\eta, \xi)=\sum_{|\alpha|,|\beta|=m} \sum_{|k| \leqslant J} a_{\alpha \beta}^{k} \exp (i k \eta) \xi^{\alpha+\beta} \quad\left(\eta \in \mathbb{R}, \xi \in \mathbb{R}^{n}\right) .
$$

Здесь переменная $\xi \in \mathbb{R}^{n}$, отвечающая дифференцированию и переменная $\eta \in \mathbb{R}$, отвечающая сдвигу, изменяются независимо. Обычным же символом будет $a_{R}\left(\varepsilon \xi_{1}, \xi\right)$.

Теорема 1 (см. [5, теорема 2.13]). Уравнение (1) является сильно эллиптическим в $\bar{\Omega}$ для любого значения параметра $\varepsilon>0$ тогда и только тогда, когда

$$
\operatorname{Re} a_{R}(\eta, \xi)>0 \quad\left(\eta \in \mathbb{R}, 0 \neq \xi \in \mathbb{R}^{n}\right)
$$

Следующее утверждение является обобщением предыдущей теоремы на случай произвольной ограниченной области $Q \subset \mathbb{R}^{n}$ с кусочно гладкой границей.

Утверждение 1. Уравнение (1) является сильно эллиптическим в $\bar{Q}$ для любого значения параметра $\varepsilon>0$ тогда и только тогда, когда выполнено неравенство (5). 
Доказательство. Достаточность. Пусть неравенство (5) выполнено. Для ограниченной области $Q$ найдется цилиндр $\Omega_{M}$, такой, что $Q \subset \Omega_{M}$. Пусть $u \in C_{0}^{\infty}(Q)$ - произвольная функция (продолженная нулем вне $Q$ ). Тогда $u \in C_{0}^{\infty}\left(\Omega_{M}\right)$ и в силу теоремы 1 неравенство Гординга выполнено для уравнения $(2)$ в области $\Omega_{M}$, следовательно, и в $Q$.

Необходимость. Для области $Q$ найдется такой цилиндр $\Omega_{m}$, что $\Omega_{m} \subset Q$. Пусть неравенство Гординга выполнено в области $Q$ для любой функции $u \in C_{0}^{\infty}(Q)$, а следовательно, и для любой функции $u \in C_{0}^{\infty}\left(\Omega_{m}\right)$. В силу теоремы 1 выполнено неравенство (5).

Введем обозначение

$$
a_{\min }:=\inf _{\eta, \xi} \operatorname{Re} a_{R}(\eta, \xi) \quad(\eta \in \mathbb{R},|\xi|=1) .
$$

Из доказательства в [5, теорема 2.13] получаем также следующее утверждение.

Утверждение 2. Для дифференииально-разностного уравнения (1) имеет место неравенcm80

$$
\operatorname{Re} \sum_{|\alpha|,|\beta|=m}\left(R_{\alpha \beta} D^{\beta} u, D^{\alpha} u\right)_{L_{2}(Q)} \geqslant a_{\min }\|u\|_{H^{m}(Q)}^{2} \quad\left(\forall u \in C_{0}^{\infty}(Q)\right),
$$

где $a_{\min }$ определено формулой (6).

3. Сильная эллиптичность возмущенных дифференциально-разностных уравнений. Рассмотрим краевую задачу для дифференциально-разностного уравнения

$$
\begin{gathered}
-\sum_{i, j=1}^{n} \frac{\partial}{\partial x_{i}}\left(R_{i j \varepsilon}^{a} \frac{\partial u(x)}{\partial x_{j}}\right)-\sum_{i}^{n} \frac{\partial}{\partial x_{i}}\left(R^{b} \frac{\partial u(x)}{\partial x_{i}}\right)=f(x) \quad(x \in Q), \\
u(x)=0 \quad(x \notin Q),
\end{gathered}
$$

где $Q$ - ограниченная область в $\mathbb{R}^{n}$ с кусочно гладкой границей, $f \in L_{2}(Q)$. Разностные операторы $R_{i j \varepsilon}^{a}: L_{2}\left(\mathbb{R}^{n}\right) \rightarrow L_{2}\left(\mathbb{R}^{n}\right), R^{b}: L_{2}\left(\mathbb{R}^{n}\right) \rightarrow L_{2}\left(\mathbb{R}^{n}\right)$ имеют вид

$$
\begin{gathered}
R_{i j \varepsilon}^{a} u(x)=a_{i j 0} u(x)+\sum_{k=1}^{J} a_{i j k}\left(u\left(x_{1}+k \varepsilon, x_{2}, \ldots, x_{n}\right)+u\left(x_{1}-k \varepsilon, x_{2}, \ldots, x_{n}\right)\right) \\
\quad\left(\varepsilon>0, a_{i j k} \in \mathbb{R}, a_{i j k}=a_{j i k}\right), \\
R^{b} u(x)=b_{0} u(x)+\sum_{h \in M \backslash 0} b_{h}(u(x+h)+u(x-h)) \quad\left(b_{h} \in \mathbb{R}\right) .
\end{gathered}
$$

Здесь $M \in \mathbb{R}^{n}$-множество векторов с целочисленными координатами, $\varepsilon>0$-малый параметр (далее $\varepsilon \rightarrow 0$ ), операторы $R_{i j \varepsilon}^{a}$ - переменные, оператор $R^{b}$ - стационарный. В операторе $R^{b}$ произвольные пространственные сдвиги, в операторе $R_{i j \varepsilon}^{a}-$ сонаправленные (без ограничения общности - в направлении оси $x_{1}$ ). Решением краевой задачи (8), (9) будем называть функцию $u \in \stackrel{\circ}{H}^{1}(Q)$, для учета однородных краевых условий продолженную нулем вне $Q$, если для любой $v \in \stackrel{\circ}{H}^{1}(Q)$ выполнено интегральное тождество

$$
\sum_{i, j=1}^{n}\left(R_{i j \varepsilon}^{a} u_{x_{j}}, v_{x_{i}}\right)_{L_{2}(Q)}+\sum_{i=1}^{n}\left(R^{b} u_{x_{i}}, v_{x_{i}}\right)_{L_{2}(Q)}=(f, v)_{L_{2}(Q)}, \quad f \in L_{2}(Q) .
$$

Здесь $\stackrel{\circ}{H}^{1}(Q)$ - пространство Соболева функций $v \in H^{1}(Q)$, у которых $\left.v\right|_{\partial Q}=0$, где равенство понимается в смысле следов. В пространстве $\stackrel{\circ}{H}^{1}(Q)$ будем использовать эквивалентное скалярное произведение

$$
(u, v)_{H^{1}(Q)}=\int_{Q} \nabla u \nabla \bar{v} d x .
$$

Введем симметричные билинейные формы $a^{\varepsilon}[u, v], b[u, v]$ :

$$
a^{\varepsilon}[u, v]:=\sum_{i, j=1}^{n}\left(R_{i j \varepsilon}^{a} u_{x_{j}}, v_{x_{i}}\right)_{L_{2}(Q)}, \quad b[u, v]:=\sum_{i=1}^{n}\left(R^{b} u_{x_{i}}, v_{x_{i}}\right)_{L_{2}(Q)} .
$$


С учетом этих обозначений интегральное тождество (11) примет вид

$$
a^{\varepsilon}[u, v]+b[u, v]=(f, v)_{L_{2}(Q)} .
$$

Получим условия равномерной (по $\varepsilon>0$ ) сильной эллиптичности уравнений $(12)$. Неравенство Гординга для него примет вид

$$
a^{\varepsilon}[u, u]+b[u, u] \geqslant c_{1}\|u\|_{H^{1}(Q)}^{2}, \quad \forall u \in C_{0}^{\infty}(Q), c_{1}>0 .
$$

Если в уравнении $(12)$ положить $b[u, v]=0$, получим

$$
a^{\varepsilon}[u, v]=(f, v)_{L_{2}(Q)} .
$$

Уравнение (14) является частным случаем уравнения вида (1).

Символ $a_{R}(\eta, \xi)$, определенный формулой (4), для уравнения (14) примет вид

$$
a_{R}(\eta, \xi)=\sum_{i, j=1}^{n}\left(a_{i j}^{0}+2 \sum_{k=1}^{J} a_{i j}^{k} \cos (k \eta)\right) \xi_{i} \xi_{j} \quad\left(\eta \in \mathbb{R}, \xi \in \mathbb{R}^{n}\right) .
$$

Введем обозначение

$$
a_{\min }:=\inf _{\eta, \xi} \operatorname{Re} a_{R}(\eta, \xi) \quad(\eta \in \mathbb{R},|\xi|=1) .
$$

В силу утверждения 2 для любого $\varepsilon>0$

$$
a^{\varepsilon}[u, u] \geqslant a_{\min }\|u\|_{H^{1}(Q)}^{2}, \quad \forall u \in C_{0}^{\infty}(Q) .
$$

Для выполнения неравенства Гординга для уравнения (14) при любых $\varepsilon>0$ в силу утверждения 1 необходимо и достаточно, чтобы $a_{\min }>0$. При этом $c_{1}=a_{\min }$ и не зависит от $\varepsilon$.

Получим теперь условия сильной эллиптичности семейства уравнений (8). Введем контрольный разностный оператор $R^{C}: L_{2}\left(\mathbb{R}^{n}\right) \rightarrow L_{2}\left(\mathbb{R}^{n}\right)$

$$
R^{C} u(x)=a_{\min } u(x)+R^{b} u(x)=a_{\min } u(x)+b_{0} u(x)+\sum_{h \in M \backslash 0} b_{h}(u(x+h)+u(x-h)) .
$$

Введем оператор $R_{Q}^{C}=P_{Q} R^{C} I_{Q}: L_{2}(Q) \rightarrow L_{2}(Q)$, где $I_{Q}: L_{2}(Q) \rightarrow L_{2}\left(\mathbb{R}^{n}\right)$ - оператор продолжения функции из $L_{2}(Q)$ нулем в $\mathbb{R}^{n}, P_{Q}: L_{2}\left(\mathbb{R}^{n}\right) \rightarrow L_{2}(Q)$ - оператор сужения функции из $L_{2}\left(\mathbb{R}^{n}\right)$ на $Q$.

Теорема 2. Уравнение (8) является сильно эллиптическим в $\bar{Q}$ для любого значения параметра $\varepsilon>0$ тогда и только тогда, когда контрольный оператор $R_{Q}^{C}$ является положительно определенным.

Доказательство. Достаточность. Пусть оператор $R_{Q}^{C}$ является положительно определенным, т.е.

$$
\left(R_{Q}^{C} u, u\right)_{L_{2}(Q)} \geqslant c_{1}(u, u)_{L_{2}(Q)} \quad\left(c_{1}>0, \forall u \in C_{0}^{\infty}(Q)\right) .
$$

Докажем, что для всех $\varepsilon>0$ справедлива формула (13) с той же константой $c_{1}$ :

$$
\sum_{i=1}^{n}\left(R_{Q}^{C} u_{x_{i}}, u_{x_{i}}\right)_{L_{2}(Q)} \geqslant c_{1} \sum_{i=1}^{n}\left(u_{x_{i}}, u_{x_{i}}\right)_{L_{2}(Q)}=c_{1}\|u\|_{H^{1}(Q)}^{2} \quad\left(\forall u \in C_{0}^{\infty}(Q)\right) .
$$

Отсюда

$$
b[u, u]+a_{\min }\|u\|_{H^{1}(Q)}^{2}=\sum_{i=1}^{n}\left(R_{Q}^{C} u_{x_{i}}, u_{x_{i}}\right)_{L_{2}(Q)} \geqslant c_{1}\|u\|_{H^{1}(Q)}^{2} \quad\left(\forall u \in C_{0}^{\infty}(Q)\right) .
$$

Следовательно, из формул (16), (19)

$$
a^{\varepsilon}[u, u]+b[u, u]=\left(a^{\varepsilon}[u, u]-a_{\min }\|u\|_{H^{1}(Q)}^{2}\right)+\left(a_{\min }\|u\|_{H^{1}(Q)}^{2}+b[u, u]\right) \geqslant c_{1}\|u\|_{H^{1}(Q)}^{2} .
$$

Отсюда для всех $\varepsilon>0$ следует условие сильной эллиптичности (13) с константой $c_{1}$ из неравенства (18). 
Необходимость. Предположим противное. Пусть оператор $R_{Q}^{C}$ не является положительно определенным. Используем формализм, построенный А. Л. Скубачевским в [7]. Для оператора $R_{Q}^{C}$ построим разбиение $\Re$ области $Q$ на непересекающиеся подобласти $Q_{r}(r=1,2, \ldots): \bigcup_{r} \bar{Q}_{r}=\bar{Q}$ и для любых $Q_{r_{1}}$ и $h \in\{M,-M\}$ или найдется такое $Q_{r_{2}}$, что $Q_{r_{2}}=Q_{r_{1}}+h$, или $Q_{r_{1}}+h \in \mathbb{R}^{n} \backslash Q$. Здесь $M-$ множество векторов из формулы (10).

Разбиению $\Re$ поставим в соответствие граф. Вершины графа это подобласти $Q_{r}$, ребра графасдвиги $h \in\{M,-M\}$. Если $Q_{r_{2}}=Q_{r_{1}}+h$, то вершины графа, ассоциированные с подобластями $Q_{r_{2}}, Q_{r_{1}}$ связаны ребром $h$. Введем на множестве подобластей (вершин графа) отношение эквивалентности: подобласти $Q_{r_{1}}, Q_{r_{2}} \in \Re$ принадлежат к одному классу, если существует путь в графе из вершины $Q_{r_{1}}$ в $Q_{r_{2}}$. Это отношение порождает разбиение $\Re$ на непересекающиеся классы. Обозначим подобласти $Q_{s l}$, где $s$ номер класса и $l$ номер области в этом классе. Каждый класс $s$ состоит из конечного числа $N=N(s)$ подобластей $Q_{s l}$.

Обозначим через $L_{2}\left(\bigcup_{l} Q_{s l}\right)$ подпространство функций из $L_{2}(Q)$, обращающихся в нуль вне $L_{2}\left(\bigcup_{l} Q_{s l}\right)(l=1, \ldots, N(s))$.

Обозначим через $P_{s}: L_{2}(Q) \rightarrow L_{2}\left(\bigcup_{l} Q_{s l}\right)$ оператор ортогонального проектирования на $L_{2}\left(\bigcup_{l} Q_{s l}\right)$. В силу [7, лемма 8.5] $L_{2}\left(\bigcup_{l} Q_{s l}\right)$ является инвариантным подпространством оператора $R_{Q}^{C}$, при этом $L_{2}(Q)=\bigoplus_{s} L_{2}\left(\bigcup_{l} Q_{s l}\right)$.

Введем изоморфизм гильбертовых пространств $U_{s}: L_{2}\left(\bigcup_{l} Q_{s l}\right) \rightarrow L_{2}^{N}\left(Q_{s 1}\right)$ по формуле

$$
\left(U_{s} u\right)_{l}(x)=u\left(x+h_{s l}\right) \quad\left(x \in Q_{s 1}\right),
$$

где $l=1, \ldots, N=N(s), h_{s l}$ таково, что $Q_{s 1}+h_{s l}=Q_{s l}\left(h_{s 1}=0\right)$,

$$
L_{2}^{N}\left(Q_{s 1}\right)=\prod_{l=1}^{N} L_{2}\left(Q_{s 1}\right) .
$$

В силу [7, лемма 8.6] оператор $R_{Q s}: L_{2}^{N}\left(Q_{s 1}\right) \rightarrow L_{2}^{N}\left(Q_{s 1}\right)$, заданный формулой $R_{Q s}=U_{s} R_{Q} U_{s}^{-1}$, есть оператор умножения на матрицу $R_{s}=R_{s}(x)\left(x \in Q_{s 1}\right)$ порядка $N(s) \times N(s)$, элементы матрицы вычисляются по формуле

$$
r_{i j}^{s}(x)= \begin{cases}b_{h}, & h=h_{s j}-h_{s i} \in M, \\ 0, & h_{s j}-h_{s i} \notin M .\end{cases}
$$

При этом

$$
\left(R_{Q}^{C} w, v\right)_{L_{2}\left(\bigcup_{l} Q_{s l}\right)}=\left(R_{s} W, V\right)_{L_{2}^{N}\left(Q_{s 1}\right)},
$$

$v, w \in L_{2}\left(\bigcup_{l} Q_{s l}\right) ; V=U_{s} v, W=U_{s} w \in L_{2}^{N}\left(Q_{s 1}\right)$.

Обозначим через $n_{1}$ число различных матриц $R_{s_{\nu}}\left(\nu=1, \ldots, n_{1}\right)$.

В силу [7, лемма 8.8] оператор $R_{Q}^{C}$ положительно определен тогда и только тогда, когда все матрицы $R_{s_{\nu}}\left(\nu=1, \ldots, n_{1}\right)$ положительно определены. Отсюда, так как $R_{Q}^{C}$ не является положительно определенным, найдется класс $s$ и семейство $\left\{Q_{s k}\right\}_{k=1}^{N}$ областей разбиения области $Q$ и матрица $R_{s}^{b}$ с неположительным собственным значением $\lambda_{b} \leqslant 0$ и собственным вектором $q=\left(q_{1}, \ldots, q_{N}\right) \in \mathbb{R}^{N}: R_{s}^{b} q=\lambda_{b} q$. Здесь $N$ - размерность матрицы $R_{s}^{b}$ и количество подобластей в классе $s$. Пространство

$$
C_{0}^{\infty}\left(\bigcup_{k=1}^{N} Q_{s k}\right)
$$

инвариантно для оператора $R_{Q}^{C}$ и действие этого оператора на нем сводится к умножению на матрицу $R_{s}^{b}$, заданную формулой $(21)$. Для билинейной формы $\tilde{a}^{\varepsilon}[u, v]:=a^{\varepsilon}[u, v]-a_{\min }(u, v)_{\dot{H}^{1}(Q)}$ символ $\tilde{a}_{R}(\eta, \xi)$ примет вид

$$
\tilde{a}_{R}(\eta, \xi)=a_{R}(\eta, \xi)-a_{\min }|\xi|^{2} \quad\left(\eta \in \mathbb{R}, \xi \in \mathbb{R}^{n}\right) .
$$


Из формулы (23) следует, что

$$
\min _{\eta, \xi} \tilde{a}_{R}(\eta, \xi)=0 \quad\left(\eta \in \mathbb{R}, \xi \in \mathbb{R}^{n},|\xi|=1\right) .
$$

В силу утверждения 1 уравнение, соответствующее интегральному тождеству

$$
\tilde{a}^{\varepsilon}[u, v]=(f, v)_{L_{2}(Q)}
$$

для любой области $G \subset \mathbb{R}^{n}$ не является равномерно по $\varepsilon>0$ сильно эллиптическим, т.е. для любого $\delta>0$ найдутся такие $\varepsilon(\delta)>0$ и функция $u_{\varepsilon} \in C_{0}^{\infty}(G)$, что

$$
\tilde{a}^{\varepsilon}\left[u_{\varepsilon}, u_{\varepsilon}\right]_{G}<\delta\left\|u_{\varepsilon}\right\|_{H^{1}(G)}^{2} .
$$

Здесь $\tilde{a}^{\varepsilon}\left[u_{\varepsilon}, u_{\varepsilon}\right]_{G}-$ сужение формы $\tilde{a}^{\varepsilon}\left[u_{\varepsilon}, u_{\varepsilon}\right]$ на область $G$. В качестве области $G$ в формуле $(24)$ возьмем область $G_{s 1} \subset Q_{s 1}$. При этом выберем $\varepsilon$ достаточно малым, чтобы для любой функции $u_{\varepsilon}(x) R_{i j \varepsilon}^{a} u_{\varepsilon}(x) \in C_{0}^{\infty}\left(Q_{s 1}\right)$, т.е. разностные операторы $R_{i j \varepsilon}^{a}$ не сдвигают носитель функции $u_{\varepsilon}(x) \in$ $C_{0}^{\infty}\left(G_{s 1}\right)$ за пределы области $Q_{s 1}: \operatorname{supp}\left(u_{\varepsilon}(x)\right) \subset G_{s 1} \Rightarrow \operatorname{supp}\left(R_{i j \varepsilon}^{a} u_{\varepsilon}(x)\right) \subset Q_{s 1}$.

Построим функцию $V \in L_{2}^{N}\left(Q_{s 1}\right), V(x)=u_{\varepsilon}(x) q=\left(u_{\varepsilon}(x) q_{1}, \ldots, u_{\varepsilon}(x) q_{N}\right)$. Введем обозначение

$$
Q_{s}^{N}=\bigcup_{l=1}^{N} Q_{s l}
$$

Функции $V$ соответствует $v \in L_{2}\left(Q_{s}^{N}\right), v=U_{s}^{-1}(V)$, где $U_{s}$ - изометрический изоморфизм, определенный формулой $(20):(v, v)_{L_{2}\left(Q_{s}^{N}\right)}=(V, V)_{L_{2}^{N}\left(Q_{s 1}\right)}$. Области $G_{s l} \subset Q_{s l}$ получаются соответствующими сдвигами области $G_{s 1}$. Введем обозначение

$$
G_{s}^{N}=\bigcup_{l=1}^{N} G_{s l}
$$

По построению $\operatorname{supp}(v) \subset G_{s}^{N}$ и в силу $(24)$, примененному к области $G_{s 1}$,

$$
\tilde{a}^{\varepsilon}[v, v]_{Q}=\tilde{a}^{\varepsilon}[v, v]_{G_{s}^{N}}=\sum_{l=1}^{N} q_{l}^{2} \tilde{a}^{\varepsilon}\left[u_{\varepsilon}, u_{\varepsilon}\right]_{G_{s 1}}<\delta|q|^{2}\left(u_{\varepsilon}, u_{\varepsilon}\right)_{\stackrel{\circ}{1}^{1}\left(G_{s 1}\right)}=\delta(v, v)_{\dot{H}^{1}(Q)} .
$$

С учетом (22), поскольку $q$ - собственный вектор матрицы $R_{s}^{b}$ :

$$
\begin{array}{r}
\tilde{b}[v, v]_{Q}=\sum_{i=1}^{n}\left(R_{Q}^{C} v_{x_{i}}, v_{x_{i}}\right)_{L_{2}(Q)}=\sum_{i=1}^{n}\left(R_{Q}^{C} v_{x_{i}}, v_{x_{i}}\right)_{L_{2}\left(Q_{s}^{N}\right)}=\sum_{i=1}^{n}\left(R_{s}^{b} V_{x_{i}}, V_{x_{i}}\right)_{L_{2}^{N}\left(Q_{s 1}\right)}= \\
=\sum_{i=1}^{n}\left(R_{s}^{b} q\left(u_{\varepsilon}\right)_{x_{i}}, q\left(u_{\varepsilon}\right)_{x_{i}}\right)_{L_{2}^{N}\left(Q_{s 1}\right)}=\sum_{i=1}^{n}\left(\lambda_{b} q\left(u_{\varepsilon}\right)_{x_{i}}, q\left(u_{\varepsilon}\right)_{x_{i}}\right)_{L_{2}^{N}\left(Q_{s 1}\right)}=\lambda_{b} \sum_{l=1}^{N} q_{l}^{2}\left(u_{\varepsilon}, u_{\varepsilon}\right)_{\dot{H}^{1}\left(G_{s 1}\right)}= \\
=\lambda_{b}|q|^{2}\left\|u_{\varepsilon}\right\|_{\dot{H}^{1}\left(G_{s 1}\right)}^{2}=\lambda_{b}(v, v)_{\dot{H}^{1}(Q)} .
\end{array}
$$

Отсюда получаем

$$
\tilde{a}^{\varepsilon}[v, v]_{Q}+\tilde{b}[v, v]_{Q}<\left(\delta+\lambda_{b}\right)(v, v)_{\dot{H}^{1}(Q)} \leqslant \delta(v, v)_{\dot{H}^{1}(Q)},
$$

и уравнение (12) не является равномерно по $\varepsilon$ сильно эллиптическим. Теорема доказана.

Пример 1. Рассмотрим задачу

$$
\begin{gathered}
-\Delta\left(R_{\varepsilon}^{a} u(x)+R^{b} u(x)\right)=f(x) \quad(x \in Q), \\
u(x)=0 \quad(x \notin Q) .
\end{gathered}
$$

Разностные операторы заданы формулой (10). Для уравнения (25) неравенство Гординга примет вид

$$
\sum_{i=1}^{n}\left(R_{\varepsilon}^{a} u_{x_{i}}, u_{x_{i}}\right)_{L_{2}(Q)}+\left(R^{b} u_{x_{i}}, u_{x_{i}}\right)_{L_{2}(Q)} \geqslant c_{1}\|u\|_{H^{1}(Q)}^{2} \quad \forall u \in C_{0}^{\infty}(Q), c_{1}>0 .
$$


Символ $a_{R}(\eta, \xi)$, определенный формулой (15), для уравнения (25) примет вид

$$
a_{R}(\eta, \xi)=\sum_{i=1}^{n}\left(a^{0}+2 \sum_{k=1}^{J} a^{k} \cos (k \eta)\right) \xi_{i}^{2}=\left(a^{0}+2 \sum_{k=1}^{J} a^{k} \cos (k \eta)\right)|\xi|^{2} \quad\left(\eta \in \mathbb{R}, \xi \in \mathbb{R}^{n}\right) .
$$

Введем обозначение $g(\eta)=a_{R}(\eta, \xi)\left(\eta \in \mathbb{R}, \xi \in \mathbb{R}^{n},|\xi|=1\right)$. Тогда

$$
g(\eta)=a_{0}+2 \sum_{k=1}^{J} a^{k} \cos (k \eta) \quad(\eta \in \mathbb{R}) .
$$

Неравенство Гординга для оператора $-\Delta\left(R_{\varepsilon}^{a} u(x)\right)$ имеет вид

$$
\sum_{i=1}^{n}\left(R_{\varepsilon}^{a} u_{x_{i}}, u_{x_{i}}\right)_{L_{2}(Q)} \geqslant c_{1}\|u\|_{H^{1}(Q)}^{2} \quad \forall u \in C_{0}^{\infty}(Q), c_{1}>0 .
$$

Введем обозначение $a_{\min }:=\min _{\eta} g(\eta)$. В силу утверждения 1 для сильной эллиптичности семейства операторов $-\Delta\left(R_{\varepsilon}^{a} u(x)\right)$ необходимо и достаточно $a_{\min }>0$. При этом $c_{1}=a_{\min }$ и не зависит от $\varepsilon$.

Введем оператор $R^{C}:=R^{b}+a_{\min } I, R_{Q}^{C}=P_{Q} R^{C} I_{Q}$, где $I$ - тождественный оператор. В силу теоремы 2 для выполнения неравенства (27) необходимо и достаточно, чтобы оператор $R_{Q}^{C}$ был положительно определен.

4. Переход к предельной задаче. Наряду с задачей (8), (9) рассмотрим предельную краевую задачу

$$
\begin{gathered}
-\sum_{i, j=1}^{n} \frac{\partial}{\partial x_{i}}\left(a_{i j} \frac{\partial u(x)}{\partial x_{j}}\right)-\sum_{i=1}^{n} \frac{\partial}{\partial x_{i}}\left(R^{b} \frac{\partial u(x)}{\partial x_{i}}\right)=f(x) \quad(x \in Q), \\
u(x)=0 \quad(x \notin Q),
\end{gathered}
$$

где

$$
a_{i j}^{\lim }=a_{i j}^{0}+2 \sum_{k=1}^{J} a_{i j}^{k} .
$$

Уравнение (29) - это частный случай уравнения (8). Его символ

$$
a_{\lim }(\eta, \xi)=\sum_{i, j=1}^{n}\left(a_{i j}^{0}+2 \sum_{k=1}^{J} a_{i j}^{k}\right) \xi_{i} \xi_{j}=a_{R}(0, \xi) \quad\left(\eta \in \mathbb{R}, \xi \in \mathbb{R}^{n}\right) .
$$

Следовательно, предельное уравнение (29) является сильно эллиптическим, если оператор $R_{Q}^{C}$ является положительно определенным.

Теорема 3. Пусть оператор $R_{Q}^{C}$ является положительно определенным. Тогда для любой функици $f \in L_{2}(Q)$ и любом фиксированном значении параметра $\varepsilon>0$ краевая задача (8), (9) имеет единственное решение $u_{\varepsilon} \in \stackrel{\circ}{H}^{1}(Q)$. При этом $\left\|u_{\varepsilon}-u_{\lim }\right\|_{\AA^{1}(Q)} \rightarrow 0, \varepsilon \rightarrow 0$, где $u_{\lim } \in \stackrel{\circ}{H}^{1}(Q)$ - решение предельной задачи (29), (30).

Доказательство аналогично доказательству [5, следствие 2.4].

Пример 2. Рассмотрим задачу (25), (26) из примера 1 для круга $Q \subset \mathbb{R}^{2}$ единичного радиуса, $x=\left(x_{1}, x_{2}\right)$, разностные операторы

$$
\begin{gathered}
R^{b} u(x)=b_{0} u\left(x_{1}, x_{2}\right)+b_{1}\left(u\left(x_{1}+1, x_{2}\right)+u\left(x_{1}-1, x_{2}\right)\right)+b_{2}\left(u\left(x_{1}, x_{2}+1\right)+u\left(x_{1}, x_{2}-1\right)\right), \\
R_{\varepsilon}^{a} u(x)=a_{0} u\left(x_{1}, x_{2}\right)+a_{1}\left(u\left(x_{1}+\varepsilon, x_{2}\right)+u\left(x_{1}-\varepsilon, x_{2}\right)\right), \quad \varepsilon>0 .
\end{gathered}
$$

В силу (28) символ $g(\eta)=a_{0}+2 a_{1} \cos (\eta)(\eta \in \mathbb{R})$. Тогда $a_{\min }=a_{0}-2\left|a_{1}\right|$. Контрольный разностный оператор имеет вид

$R^{C} u(x)=\tilde{b}_{0} u\left(x_{1}, x_{2}\right)+b_{1}\left(u\left(x_{1}+1, x_{2}\right)+u\left(x_{1}-1, x_{2}\right)\right)+b_{2}\left(u\left(x_{1}, x_{2}+1\right)+u\left(x_{1}, x_{2}-1\right)\right), \quad \tilde{b}_{0}=b_{0}+a_{\min }$. 


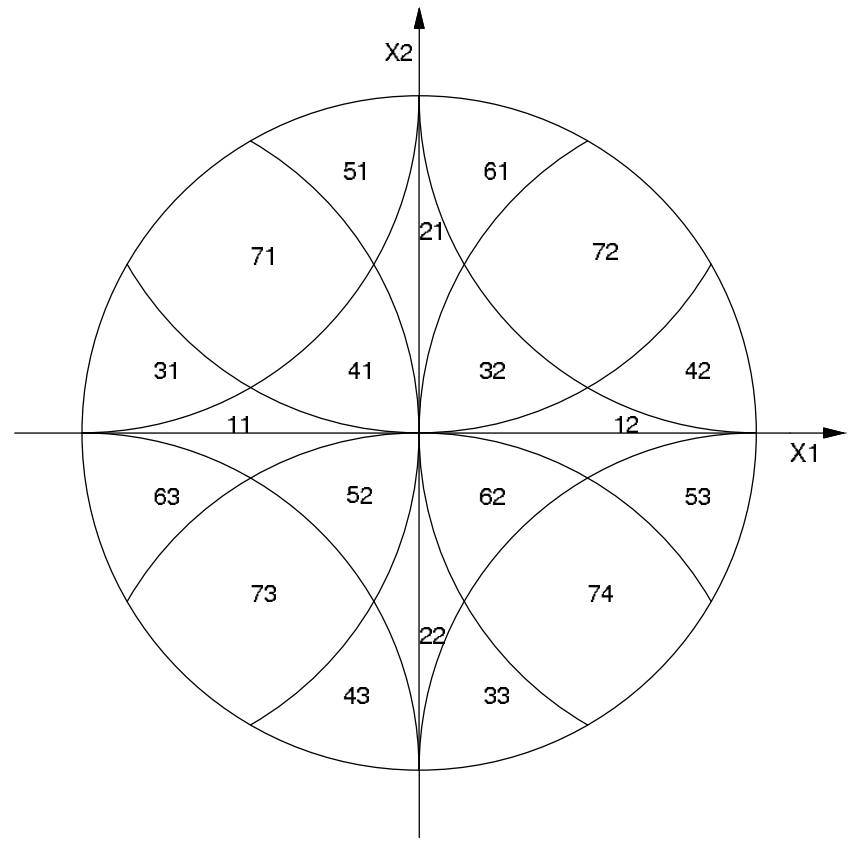

Рис. 1

В силу теоремы 2 критерием сильной эллиптичности уравнений (25) для всех $\varepsilon>0$ является положительная определенность оператора $R_{Q}^{C}=P_{Q} R^{C} I_{Q}$. Разбиение области $Q$ для разностного оператора $R_{Q}^{C}$ состоит из семи классов подобластей. Эти подобласти изображены на рис. 1 . На $s$ м классе действию разностного оператора $R_{Q}^{C}$ соответствует умножение на матрицу $R_{s}(s=$ $1, \ldots, 7)$ :

$$
R_{1}=\left(\begin{array}{cc}
\tilde{b}_{0} & b_{1} \\
b_{1} & \tilde{b}_{0}
\end{array}\right), \quad R_{2}=\left(\begin{array}{cc}
\tilde{b}_{0} & b_{2} \\
b_{2} & \tilde{b}_{0}
\end{array}\right), \quad R_{3-6}=\left(\begin{array}{ccc}
\tilde{b}_{0} & b_{1} & 0 \\
b_{1} & \tilde{b}_{0} & b_{2} \\
0 & b_{2} & \tilde{b}_{0}
\end{array}\right), \quad R_{7}=\left(\begin{array}{cccc}
\tilde{b}_{0} & b_{1} & b_{2} & 0 \\
b_{1} & \tilde{b}_{0} & 0 & b_{2} \\
b_{2} & 0 & \tilde{b}_{0} & b_{1} \\
0 & b_{2} & b_{1} & \tilde{b}_{0}
\end{array}\right) .
$$

Необходимым и достаточным условием положительной определенности $R_{Q}^{C}$ является положительная определенность этих матриц. Нетрудно убедиться, что эти матрицы положительно определены при выполнении условий

$$
\tilde{b}_{0}>0, \quad\left|b_{1}\right|<\tilde{b}_{0}, \quad\left|b_{2}\right|<\tilde{b}_{0}, \quad b_{1}^{2}+b_{2}^{2}<\tilde{b}_{0}^{2}, \quad\left|b_{1}\right|+\left|b_{2}\right|<\tilde{b}_{0}
$$

и, окончательно,

$$
\left|b_{1}\right|+\left|b_{2}\right|+2\left|a_{1}\right|<b_{0}+a_{0} .
$$

При выполнении этого условия в силу теоремы 3 уравнение $(25)$ является сильно эллиптическим для всех $\varepsilon>0$, решения $u_{\varepsilon} \in \stackrel{\circ}{H}^{1}(Q)$ задач $(25),(26)$ существуют и $\left\|u_{\varepsilon}-u_{\text {lim }}\right\|_{H^{1}(Q)} \rightarrow 0, \varepsilon \rightarrow 0$, где $u_{\lim } \in \stackrel{\circ}{H}^{1}(Q)$ - решение предельной задачи

$$
\begin{gathered}
-\Delta\left(R_{\lim }^{b} u(x)\right)=f(x) \quad(x \in Q), \\
u(x)=0 \quad(x \notin Q)
\end{gathered}
$$

с разностным оператором

$R_{\lim }^{b} u(x)=\left(b_{0}+a_{0}+2 a_{1}\right) u\left(x_{1}, x_{2}\right)+b_{1}\left(u\left(x_{1}+1, x_{2}\right)+u\left(x_{1}-1, x_{2}\right)\right)+b_{2}\left(u\left(x_{1}, x_{2}+1\right)+u\left(x_{1}, x_{2}-1\right)\right)$.

Заметим, что условием сильной эллиптичности предельного уравнения является

$$
\left|b_{1}\right|+\left|b_{2}\right|<b_{0}+a_{0}+2 a_{1} .
$$


Автор выражает благодарность Л. Е. Россовскому и А. Л. Скубачевскому за внимание к работе и полезные обсуждения результатов.

\section{СПИСОК ЛИТЕРАТУРЫ}

1. Онанов Г. Г., Скубачевский А. Л. Дифференциальные уравнения с отклоняющи-мися аргументами в стационарных задачах механики// Прикл. мех. - 1979. - 15, № 5. - С. 30-47.

2. Скубачевский А. Л. О некоторых нелокальных эллиптических краевых задачах// Диффер. уравн. 1982. - 18, № 9. - С. 1590-1599.

3. Скубачевский А. Л. Краевые задачи для эллиптических дифференциально-разностных уравнений и их приложения// Усп. мат. наук. - 2016. - 71, № 5 (431). - С. 3--112.

4. Ivanova E. P. Continuous dependence on translations of the independent variable for solutions of boundaryvalue problems for differential-difference equations// J. Math. Sci. — 2018. - 233, № 6. - P. 828-852.

5. Rossovskii L. E. Elliptic functional differential equations with contractions and extensions of independent variables of the unknown function// J. Math. Sci. - 2017. - 223, № 4. - P. 351-493.

6. Skubachevskii A. L. The first boundary-value problem for strongly elliptic differential difference equations// J. Differ. Equations. - 1986. - 63, № 3. - P. 332-361.

7. Skubachevskii A. L. Elliptic Functional-Differential Equations and Applications. - Basel-Boston-Berlin: Birkhäuser, 1997.

8. Skubachevskii A. L. Bifurcation of periodic solutions for nonlinear parabolic functional differential equations arising in optoelectronics// Nonlin. Anal. Theory Meth. Appl. — 1998. — 32, № 2. — P. 261-278.

Иванова Елена Павловна

Московский авиационный институт

(национальный исследовательский университет);

Российский университет дружбы народов (РУДН), Москва

E-mail: elpaliv@yandex.ru 